\section{Contemporary approaches to prostate cancer management}
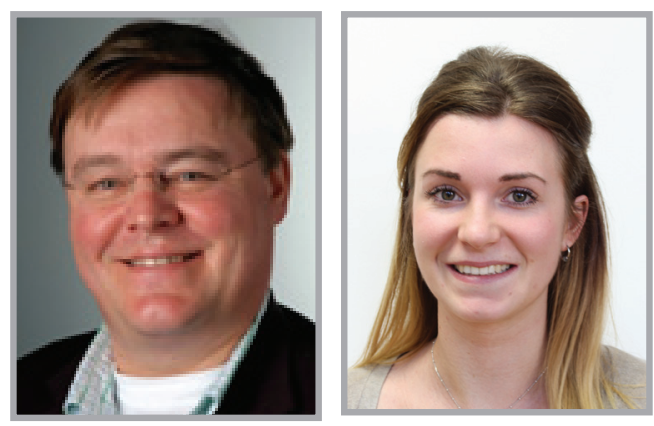

\author{
"It is clear that there is an \\ increasing role of precision \\ medicine in advanced \\ prostate cancer therapeutics.”
}

\section{Future ONCOLOGY}

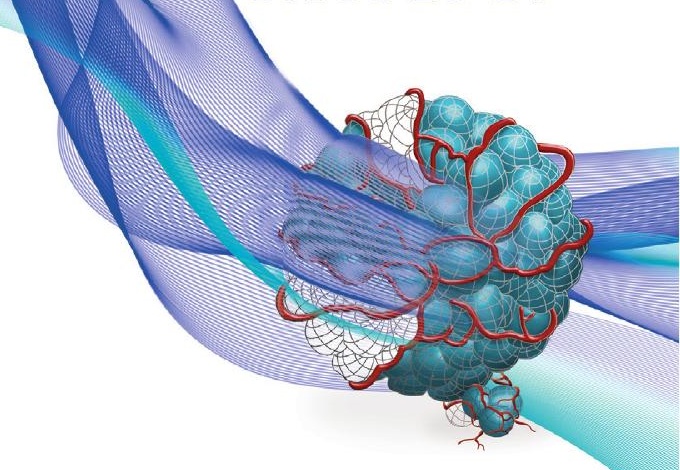

Jack A Schalken ${ }^{* 1}$ \& Gemma Westcott ${ }^{2}$
The early diagnosis of prostate cancer and the treatment of localized and advanced disease have been through an era of rapid and exciting developments. However, significant improvements are urgently needed. The limitations of the diagnostic triage that leads to the diagnosis of this disease need to be improved by the use of new biomarkers and imaging. Only when successful, we may take advantage of the potential to reduce mortality by population-based screening. More elegant and precise surgery may reduce the comorbidities associated with radical interventions. Hope is on the horizon for new therapies for metastatic prostate cancer. So far, a profound understanding of molecular endocrinology and androgen receptor signaling has led to new endocrine therapies that have the potential to influence the biology of the disease.

The early diagnosis of prostate cancer still faces the prostate-specific antigen dilemma: early detection leads to improved outcomes, but at the cost of significant overtreatment. The authors Stephan and Jung discuss the biomarkers that are emerging and are close to, or already in, clinical practice [1]. Kalikrein-derived markers assessed in serum, and cancer specific mRNA in urine. So far, Prostate Health Index (PHI) and $P C A 3$ are US FDA approved. The exact relation between urinary $P C A 3$ and indolent prostate cancer has not matured yet. In fact, the combination with gene fusions transcripts looks more promising. The positive outcome of this article is that there is clear perspective that an algorithm where a serum kalikrein-derived panel combined with urinary biomarkers, can be developed to identify patients with clinically significant prostate cancer. Just like the introduction of new surgical techniques, new biomarkers face the challenge of delivering sufficient evidence for a controlled introduction of the new biomarkers in clinical practice. Serum prostate-specific antigen by itself has been paradigm for the introduction of new biomarkers.

Next to biomarkers assessed in serum and urine, imaging can play a crucial role in identifying patients with significant prostate cancer. Wibmer reviews the significant advances of multiparametric MRI in the diagnosis of significant cancer [2]. Confirmatory studies are underway, yet there are strong indications that via the use

\section{KEYWORDS}

- biomarkers • early diagnosis

- emerging agents $\bullet$ multiparametric $\mathrm{MRI} \bullet \mathrm{PARP} \bullet$ prostate cancer

"The early diagnosis of prostate cancer and the treatment of localized and advanced disease have been through an era of rapid and exciting developments." 
“The early diagnosis of prostate cancer still faces the prostate-specific antigen dilemma: early detection leads to improved outcomes, but at the cost of significant overtreatment." of improved imaging, the early diagnosis will be more accurate. Additionally in the more advanced stages of PrCa MRI has significant potential to detect local recurrences and whole body MRI can be useful for detecting metastatic disease.

One emerging approach for risk stratification in prostate cancer is through field carcinogenesis. Roy and colleagues disucss their work using partial wave spectroscopic microscopy to identify disorder in nano-architectural cell structures during neoplastic transformation [3]. As an advancing approach, field carcinogenesis could eventually be combined with other biomarkers, for example, miRNAs, free PSA and PCA3, to improve prostate cancer prognostication.

Gandanglia and colleagues present a well-balanced evaluation of the introduction of robotassisted radical prostatectomy in the treatment of localized prostate cancer [4]. It is interesting that a new surgical technique has been not only introduced, but also spread quickly, despite a lack of level one evidence. This would be unheard of for new pharmacological interventions or biomarkers. It is understandable though, considering the complexity of randomized studies for a new surgical technology that is already available and marketed extensively. This review shows that it is a safe technique, with apparent outcome benefits in the hands of an experienced surgeon with significant increased costs.
Finally, in a comprehensive review by Sonpavde and colleagues, the authors discuss emerging agents for metastatic castration-resistant prostate cancer and their clinical development [5]. Recently approved agents in USA, including sipuleucel-T, enzalutamide, abiraterone acetate, cabazitaxel and radium-223, extend median survival by $2-4$ months, yet there is still a need to reduce cross-resistance and advance existing therapies. Sonpavde reviews some of the most novel targets and therapeutic agents for advanced prostate cancer, including: hormonal manipulations, small molecule inhibitors of proliferation, apoptosis promoters, immunotherapy, radiotherapy, bone-targeted agents and advances in chemotherapy. It is clear that there is an increasing role of precision medicine in advanced prostate cancer therapeutics.

\section{Financial \& competing interests disclosure}

JA Schalken is inventor on PCA3-related IP. His employer has licensed the IP and receives royalties; JA Schalken has been consultant for Hologic genprobe. G Westcott is an employee of Future Medicine Ltd. The authors have no other relevant affiliations or financial involvement with any organization or entity with a financial interest in or financial conflict with the subject matter or materials discussed in the manuscript apart from those disclosed.

No writing assistance was utilized in the production of this manuscript.

\section{References}

1 Stephan C, Jung K, Ralla B. Current biomarkers for diagnosing prostate cancer. Future Oncol. 11(20), 2743-2755 (2015).

2 Wibmer AG, Vargas HA, Hricak H. Role of MRI in the diagnosis and management of prostate cancer. Future Oncol. 11(20), 2757-2766 (2015).
3 Roy HK, Momi N, Backman V, Brendler CB. Harnessing novel modalities: field carcinogenesis detection for personalizing prostate cancer management. Future Oncol. 11(20), 2737-2741 (2015).

4 Gandanglia G, Montorsi F, Karakiewicz PI, Sun M. Robot-assisted radical prostatectomy in prostate cancer. Future Oncol. 11(20), 2767-2773 (2015).

5 Hathaway AR, Baker MK, Guru S. Emerging agents for the therapy of advanced prostate cancer. Future Oncol. 11(20), 2775-2787 (2015). 